\title{
Cell-based Therapies for Degenerative Disc Diseases
}

\author{
Feng-Juan Lv $^{1,2}$, Victor YL Leung ${ }^{1,2}$, Kenneth MC Cheung ${ }^{1}$
}

1. Department of Orthopaedics and Traumatology, Li Ka Shing Faculty of Medicine,

The University of Hong Kong, 21 Sassoon Road, Hong Kong SAR, China

2. HKU Shenzhen Institute of Research and Innovation, China

*Correspondence should be addressed to:

Prof. Kenneth M.C. Cheung, MD

Department of Orthopaedics and Traumatology, The University of Hong Kong Medical Centre, Queen Mary Hospital, Pokfulam Road, Pokfulam, Hong Kong SAR, China

Tel.: (852) 2255 4341, Fax: (852) 28174392

Email: cheungmc@hku.hk

Other author's email:

Feng-Juan Lv: fengjuan.lv@gmail.com

Victor Y.L. Leung: vicleung@hku.hk

\section{Acknowledgements}

This work was supported by General Funding from National Science Foundation of China (NSFC, No. 81371993), Small Project Funding of The University of Hong Kong (201409176191), and General Research Fund from Research Grants Council of Hong Kong (GRF, No. 17126615). 


\section{Abstract:}

Intervertebral discs (IVDs) degeneration are responsible for the majority of cases of low back pain, which affects millions of people worldwide. Current clinical therapies for degenerative disc diseases (DDD) involve medications or physiotherapy for mild to moderate degeneration, and surgeries for severe degeneration. However, current treatments aim to remove symptoms sometimes at the cost of mobility instead of restoring the biological function. Future therapy for disc repair is in development by biological strategies, including protein/growth factor injections, gene therapy or cell therapy approaches. Cell-based therapies provides the greatest hope, as it is a continuous source of cells and cytokine production, and the safety is superior compared to gene therapy. The choice of cell source include chondrocytes, disc cells, embryonic stem cells, hematopoietic stem cells and mesenchymal stem cells (MSCs). Results of several clinical trials suggest that MSCs is the best choice of cell source for IVD regeneration. The remaining concern is that the harsh environment of the degenerated disc may have impact on the engraftment and differentiation of implanted cells. The timing of cell implantation may also have effect on the degree of disc regeneration, which may require a earlier intervention at mild to moderate level of disc degeneration to achieve satisfactory surgical outcome.

\section{Keyword:}

Intervertebral disc; Low back pain; degenerative disc diseases; Cell therapies; Mesenchymal stem cells; 


\section{Introduction:}

Intervertebral discs (IVDs) are fibrocartilaginous tissues connecting the vertebral bodies, contributing about one-third of spinal length. IVDs are important to spinal function by providing stability while permitting motion between the vertebrae. Importantly, the complex structural features of IVDs also enable them to absorb and disperse loads from physical activities. IVD degenerates during time. Degenerative disc diseases (DDD) is a progressive disorder correlated with age. The prevalence is over $70 \%$ in the age group of 50 years or younger, and over $90 \%$ in those older than 60 (1). IVD degeneration may not necessarily cause pain. Depending on individual difference, it may sometimes be asymptomatic, but it can also be painful, which is termed discogenic pain. Discogenic low back pain accounts for $26 \%-42 \%$ of chronic LBP patients (2), which affects about $70 \%$ of the population at some point in their lives. DDD is a musculoskeletal disorder and can be indicative of severe conditions like disc herniation or prolapse, radiculopathy or spinal stenosis. It imposes an enormous socio-economic burden on the affected individual and the health care services, with increased medical expenditure and reduced productivity owing to loss of working hours.

Current clinical therapies for DDD involve symptomatic relief from pain by medications or physiotherapy for mild to moderate degeneration. For severe degeneration, surgeries such as disc arthroplasty, spinal fusion, and disc decompression are often employed as a last resort. However the effect is controversial 
(3) (4) and it may cause complications such as adjacent level disease (5) that often warrants further surgical procedures. Such treatment modalities, however, neither arrest the progression of degeneration nor restore the native functional state of the IVD. Recent years, the advances in gene delivery techniques and the identification of adult stem cells has promoted the research on the possibility to treat disc degeneration with the transplantation of viable cells, especially stem cells. Expanding knowledge has enabled diverse therapeutic development and various techniques to manipulate and direct them to specific fates has promoted a stem cell-based regenerative approach to DDD.

\section{IVD structure and Cell Biology}

A healthy adult IVD is composed of three morphologically distinct components: the central gelatinous nucleus pulposus (NP), which contains chondrocyte-like cells, surrounded by a lamellated fibrous annulus fibrosus (AF) containing fibroblast-like cells, and capped by two cartilaginous endplates (EP) that anchor into the adjacent vertebrae. The IVD is rich in extracellular matrix predominantly represented by collagen and proteoglycans with distinct differences in each compartment (6). NP is rich in aggrecan and type II collagen, while AF contains type I collagen with little proteoglycans. In fact, collagenous proteins comprise $70 \%$ of the outer annulus dry weight, but only $20 \%$ are located in the central NP, while proteoglycans make up $50 \%$ of the NP (7). Although the component of NP matrix is similar to that of articular cartilage, there is much differences in the gene expression profile and matrix synthesis 
(8). The density within the NP tissue is quite lower at around 5,000 6,000 cells $/ \mathrm{mm}^{3}$ (9) compared with 15,000 cells $/ \mathrm{mm}^{3}$ in cartilage (10). The proteoglycan : collagen ratio is also different: it is $27: 1$ in the $\mathrm{NP}$, and $2: 1$ in the cartilage (11). High proteoglycan content is a important feature of the NP. The high positive charge of proteoglycans gives the NP a high osmotic potential, which draws in water and enable the NP to absorb mechanical shock and deliver it horizontally to AF. It also inhibits the ingrowth of nerve (12). Evidence has shown that the loss of proteoglycan lead to nerve and blood vessel ingrowth in an ovine annular lesion model of experimental disc degeneration (13). Due to the above mentioned reasons, NP is the most critical component of the IVD and is the region where degenerative changes first occurs. It is also the focus of IVD regeneration researches.

\section{The Etiology of DDD}

The etiology of DDD is complicated in origin. It can include occupational exposure, mechanical influences, injury, lifestyle, and genetic pre-deposition. Moreover, strong evidences have confirmed that the disturbance in the biology of the cells in the NP, $\mathrm{AF}$ and EP, which are significantly affected by the diffusion of nutrients and oxygen from the blood vessels $(14,15)$, the soluble factors in the niche (16), the mechanical loads (17) as well as ageing or senescence $(18,19)$, plays a key role in the regulation of the IVD degeneration. However, in spite of the pre-deposition factors and secondary causes, it is believed that mechanical overload is one of the initiating factors of the disorders of the IVD. The normal adult IVD is avascular and aneural 
with the exception of the outer third of the AF. When IVD degenerates, the NP is disorganized, and the proportion and types of extraellular matrix proteins changes which makes the NP more eosinophilic. Overload leads to breakdown of the matrix of the NP with the formation of fissures that eventually extend into the AF. There is often also disruption of the collagen fiber orientation in the AF, traumatic damage to the end plate, and blood vessel (20) and nerve ingrowth (21) (22) into the inner AF and NP. Which is normally avascular and aneural.

\section{Molecular Changes in the Degenerated IVD}

Molecularly, DDD is characterized by cell death and degeneration of extracellular matrix. Normal NP cells are characterized by expression of type II collagen and proteoglycans. In disc degeneration, matrix remodeling (23) leads to an increase in collagens I and III and decreased production of aggrecan. In degeneration, the anabolic : catabolic balance is broken, there is a net increase in matrix-degrading enzyme activity over natural inhibitors of such activity, which leads to loss of disc matrix. Increased degraded fragments of proteoglycan (24) (25) and other matrix proteins (26) was reported, which has the potential to trigger further inflammatory changes (27) (summarized in (28)). Aggrecan has two cleavage sites, one acted upon by MMPs and the other by members of a group of enzymes called the ADAMs family (29). Matrix metalloproteinases (MMPs) and ADAMs have both been implicated in disc degeneration (30) (31). 
Degenerated IVDs are demonstrated to be in a chronic inflammatory state with increased expression of multiple pro-inflammatory cytokines, including interleukin 1 (IL-1), matrix metalloproteinase 10 (MMP10) (32), IL-8, tumor necrosis factor- $\alpha$ (TNF- $\alpha$ ) (33), IL-10 (34), IL-2, IL-4, IL-17, monocyte chemotactic protein-1 (MCP-1) and prostaglandin E2 (PGE2) (35,36). Of these, IL-1 is particularly interesting, because IL-1 plays more important role in mediating disc matrix degradation than TNF- $\alpha$ (16). IL-1b also leads to production of MMPs and pain mediators, such as the eicosanoid prostaglandin E2, by human IVD cells (37).

\section{Potential Developing Treatments for Disc Degeneration}

One of the problems of current treatments is their inability to repair the disc. Instead the aim of current treatments is more about removing symptoms such as pain and sometimes this is realized at the cost of mobility in the case of spinal fusion. As the future therapy for disc repair, protein/growth factor injections, gene therapy or cell therapy approaches is in continuous development.

Growth factors are small proteins with pleiotrophic effects on cells, including stimulation of cell differentiation and division. Several growth factors have been investigated for their effect on disc regeneration in vitro or in vivo. These include bone morphologic protein 2 (BMP2) (38), BMP7 (39), BMP12 (38), BMP13 (40), BMP14 (growth differentiation factor 5, GDF-5) (41), transforming growth factor beta (TGF-B) (42), Insulin-like growth factor 1 (IGF-1) (43) (44), basic fibroblast 
growth factor (bFGF) (45), tissue inhibitor of metalloproteinase (TIMPs) (46), platelet-derived growth factor (PDGF) (43) (47), and so on. They are implied with positive outcomes suggesting restoration of the degenerated NP. The effect includes increased proteoglycan content and enhanced proliferation. Noteworthy, some BMPs may increase the risk of ectopic bone formation within the IVD (48), which may potentially be inhibited by BMP13 (49).

Gene transfer involves the delivery of exogenous genes to target cells, resulting in gene expression and subsequent production of proteins. Gene therapy is one way to modify the biological activity of the IVD cells. Retrovirus mediated transfer (50) of two different exogenous genes (bacterial b-galactosidase, LacZ; and human interleukin-1 receptor antagonist, IL-1Ra) to cultured bovine intervertebral end plate cells resulted in the production of IL-1Ra in $48 \mathrm{~h}$, implicating the host tissues are capable of the synthesis of regeneration drugs. Adenoviral transduction of degenerate human NP cells with anti-catabolic enzyme, TIMP-1 or BMP-2, resulted in increased proteoglycan synthesis in vitro (46). Likewise, adenovirus mediated transfer of CTGF and TIMP-1 genes to NP cells resulted in increased proteoglycan and collagen II production (51) in rhesus monkey and rabbit animal models. In the latter study, transplantation of the modified cells back into the rabbit IVD resulted in a reversal of disc degeneration (52). Therefore, the ability of degenerate NP cells to produce the required matrix proteins could be revived through genetic modification, after which the transgenic cells can be transplanted into the degenerate IVD to restore its function. 
Growth factor therapy and gene therapy has their disadvantages. The caveat with growth factor injection is that the therapeutic effects could not last long, and repeated injection may cause damage to the AF. Another concern is that at advanced levels of disc degeneration, the endogenous cells may no longer be responsive to such stimuli or there may be insufficient number of functional cells in the IVD to begin with (53) (54). The drawbacks of gene therapies are that viral vectors are often used for gene transfer, so their safety and effectiveness needs to be thoroughly evaluated before being employed in human trials. Conversely, cell based therapies offer an attractive solution to current treatments. Cell therapy does not have the problem of short-lived effect of growth factor injections. The cells transplanted is a continuous source of cytokine production. The safety is not an issue, as transplantation study has demonstrated their safety in other tissues or organs before. The only problem of concern is the viability and proliferation of the implanted cells, whether these cells can engraft and proliferate in the harsh environment of the degenerated disc. Numerous researches has been done in this aspect. The details were summarized as below.

\section{Cell therapies for Disc Repair}

In history, the investigated choice of cell source for disc repair include chondrocytes, disc cells, embryonic stem cells, and mesenchymal stem cells. 


\section{Chondrocytes}

As adult NP cells are chondrocyte like cells, attempts have been made to regenerate the disc with cartilage cells. In a rabbit study (55), the nucleus pulposus was evacuated and replaced with tissue engineered autologous chondrocytes from auricular cartilage. The implanted cells survived well in the host for up to six months, and hyaline-like cartilage formed in the place of the NP. In a porcine model (56), allogeneic juvenile chondrocytes injection into denucleated discs demonstrated abundant cartilage formation at 3 months, and to a lesser extent at 6 and 12 months. Overall, transplantation of chondrocytes to regenerate the disc is feasible, but the generated tissue is cartilage like instead of nucleus pulposus like.

\section{IVD Cells}

Based on numerous in vitro and in vivo studies on rat, rabbit, canine, porcine, ovine, bovine and human, IVD cells, including notochordal cells and NP cells are potentially a good choice for transplantation procedures. Their ability to increase the proteoglycan content, viability upon transplantation and established protocols to expand them in vitro are advantageous for cell therapy (57) (58). For instance, implantation of autologous NP cells or entire NP retarded disc degeneration (59) (60) (61). Also, injection of immortalized human NP cells into degenerate rabbit disc preserved the matrix of NP and decelerated its degradation (58).

Despite positive results of animal studies of IVD cells, the acquisition of healthy 
donor IVD cells either allogenic or autologous has for-see difficulty. It is noteworthy though that cells harvested from a degenerated IVD may not be suitable as they exhibit senescence with an altered molecular phenotype and distinct pathology (62). Similarly, autologous cells harvested from patients who are genetically susceptible to DDD may prove inferior for cell therapy since they are already pre-disposed to degeneration (63). More importantly, harvesting adult NP or AF cells from prospective healthy discs of the patients is also counterproductive as it could result in increased morbidity or further damage to the IVD. Allogenic sources may be the alternative; but there is lack of evidence in human studies. In addition, obtaining healthy notochord cells is not a feasible option owing to the absence of such cells in adults.

\section{Embryonic Stem Cells}

Stem cells possess multi-lineage differentiation potential and self-renew capacity. Stem cells hold great promise for regenerative therapy. They are unique in that they are pluripotent (e.g. embryonic stem cells, ESCs) or multipotent (e.g. mesenchymal stem cells, MSCs), possessing the ability to self-renew as well as differentiate into specific cell lineages. They could be utilized if they could be successfully directed to a NP or AF cell fate before transplantation.

ESCs are derived from inner cell mass of blastocysts, and have an advantage over MSCs as they can give rise to cells from all three germ layers (endoderm, mesoderm 
and ectoderm) and exhibit extensive proliferation ability in vitro. In spite of this, their usage in clinical settings is hindered by a number of other factors such as: difficulties in acquisition owing to ethical concerns, immune rejection after allogeneic transplantation, risk of disease transmission and the possibility of teratoma formation. The use of ESC-derived chondro-progenitors may be an alternative option. In a rabbit study, at 8 weeks post-ESC derived chondro-progenitors implantation (64), islands of cell growth were observed.

\section{Mesenchymal Stem Cells}

MSCs is the most intensively investigated type of stem cells for intervertebral disc repair. MSCs are firstly derived in bone marrow but later found in many tissues or organs. They are characterized by their tri-potency (osteogenesis, chondrogenesis, adipogenesis) and marker expression (65). The safety and efficacy of MSC implantation for tissue regeneration has well been documented (66) (67). In vitro, MSC has been proved to be able to stimulate the proliferation, and the synthesis of collagen II and aggrecan, of cultured NP cells, in co-culture system (68) (69) (70). Numerous on-going studies by different research groups also highlighted the potential of MSCs to differentiate into NP-like phenotype (71) (72) (73) (74). In vivo, MSCs have been injected into mouse (75), rabbit (76), rat (77), canine (78), and porcine (79) IVD, with encouraging results. MSCs might be the most idea candidate of cell therapy for disc repair. This is because they have several advantages. First, They have no ethical concerns as ESCs have. Second, their safety is evidenced, they do not form 
teratoma. They are adult stem cells which means they could be harvested easily from the patient self. Allogenic transplantation is also feasible due to the capacity of MSCs to escape immune surveillance due to the lack of HLA class II antigen presentation.

\section{Published Clinical Trials}

Cell therapy for disc repair has already marched into clinical phase. The outcome of several clinical trials has been published. The utilized cell source include HSCs, IVD cells, chondrocytes, and MSCs.

The effect of HSC on disc repair is minimum. In a HSC transplantation study with 10 patients enrolled (80), no improvement was recorded in pain assessment after 1 year post transplantation. However, the cell source used in this study is of doubt. As the author described, 'A total of $5 \mathrm{cc}$ of bone marrow aspirate was obtained. Next, $1 \mathrm{cc}$ of HSCs were inserted into each of the problematic discs.' It is not clear whether the authors have purified CD34+ HSCs from the bone marrow for the transplantation, or they just injected raw bone marrow. This is different as bone marrow not only contains HSCs. It also harbors MSCs, monocytes and contains various growth factors which may potentially affect the disc. Generally, HSC may not be a suitable source of stem cells for IVD repair as it is not reported to be a type of progenitor cells to give rise to cells of mesodermal origin.

Chondrocytes has been implicated for their effect in disc repair clinically. In a US 
study (81), fifteen patients were treated with a single percutaneous delivery of $1 \sim 2 \times 10^{7} \mathrm{NuQu}$ juvenile chondrocytes with fibrin carrier. Pain and life quality, represented by the mean ODI (Oswestry Disability Index), NRS (Numerical Rating Scale), and SF-36 (36-Item Short Form Health Survey) physical component summary scores all improved significantly from baseline. At 6 months follow up, 77\% of the examined patients showed MRI improvement, and 23\% showed improvement in disc contour or height.

There is also one study documenting injection of disc cell could alleviate disc degeneration. In a Germany study, autologous disc cell transplantation (82) in 12 patients resulted in reduced pain, maintained disc height and water content at 2 years post transplantation, when compared to the control group constituted by 16 patients who received discectomy-only.

More clinical evidence support MSCs as the idea source of cell for IVD regeneration. In a Japan study (83), autologous bone marrow MSCs carried in collagen sponges was transplanted into the surgical discs of 2 patients. 2 year after the transplantation, low back pain and lower leg numbness was improved. Signal intensity on T2-weighed MRI was high, indicating high moisture content in the discs. However, the disc height has not changed and the sample size in this study was too small to confirm the long term effect of MSC transplantation clinically. Later, in another MSC transplantation study (84) in 10 patients, 9 patients achieved low back pain relief within 3 months, 
accompanied by a parallel improvement in disability and physical life quality. Similar to Yoshikawa's observation, the disc height has not improved. But the ratio of fluid content increased at 12 month post-op. The analgesic effect of treatment with MSC approached $71 \%$ efficacy, which was explained as the potential induction of anti-inflammatory cytokines by the MSCs.

It is noteworthy that the therapeutic efficacy may be closely associated with the purity of MSCs within the injected material. A recent study investigated the effect of autologous bone marrow concentrate injection into surgical discs. At 2 years post injection, there was a significant reduction in pain. However, for degeneration grade as assessed by the modified Pfirrmann scale, only $40 \%$ of the patients improved by one grade, while the remaining twelve patients maintained their previous score. The outcome of this study is not as satisfactory as the other clinical trials using MSCs. As the same study suggested that the degree of pain relief was linked to the concentration of MSC (or fibroblastic colony forming units) in the bone marrow concentrate, the less than satisfactory outcome may be partially related with the fact that MSCs only exists in the bone marrow at a very low frequency (85), so the quantity of MSCs injected in this study is far less than the dose in the other studies.

\section{Discussion}

Because man is the only obligate bipedal vertebrate, replicating human disorders of the IVD in other animals has proved problematic. The results obtained in animal 
studies may not be replicated in human. This endows importance of clinical studies for the efficacy of disc regeneration therapies.

Though chondrocytes have been proved to be effective in alleviating disc degeneration (81), using chondrocytes to repopulate the disc may be debated. In one way this may help to restore some of the disc spaces and function, in another sense this could be challenged, as the formation of hyaline cartilage instead of a gelatinous $\mathrm{NP}$ is against nature and may cause further long term problem. In theory and in practice, disc cells are a better source than chondrocytes. However based on the discussed reasons, there is difficulties and complications in the harvest of donor disc cells and the quality is a issue. ESCs derived chondro-progenitors may still have, but less, ethical issue as ESCs, besides the differentiation into NP like cells in vitro before transplantation is yet to be established. MSCs is, and will remain as, for the foreseeable future, the best choice of cell source for IVD regeneration.

Currently, the study on the utilization of MSCs in disc repair mostly focus on bone marrow derived MSCs. Nevertheless, MSCs can be isolated from many organs and tissues with distinct differences in characteristics and differentiation potential (reviewed in (86)). Bone marrow derived MSCs may not be the most superior source due to its high spontaneous osteogenic tendency (87). Instead, umbilical cord derived MSCs has been shown in our study to exert a higher regeneration effect than bone marrow derived MSCs in stimulating NP cell towards a healthier phenotype in vitro 
(88). More researches on alternative MSC sources will be beneficial to reveal the idea candidate source of MSC for IVD regeneration.

It is noteworthy that the implantation of MSCs into degenerated discs consensually generates satisfactory result on pain relief and disc moisture content, but has no effect on improving the disc height, as demonstrated in the three clinical studies above (83) (84) (85). These may be because the implanted MSCs needs time to produce swelling matrix, but the mechanical load on the human IVD is too high that the implanted MSCs can only produce enough matrix to maintain but not increase the disc height. To get a better outcome with increased disc height, injection with a appropriate scaffold may be of use to help to alleviate the mechanical load on the disc space and injected cells and allow more time and space for cell propagation and matrix production, which are important to maintain disc height. A range of injectable scaffold can be considered as they have been tested in animal studies. These include fibrin carrier (81), atelocollagen gel (89), alginate capsules (90), amalgam (91) or chitosan-glycerophosphate hydrogel (92), etc. Thermogelling and in situ-forming scaffolds may be a good choice to alleviate implant expulsion under mechanical stress; The components are temperature sensitive, they remain as flowable at around room temperature, while change into compact hydrogel at around $37^{\circ} \mathrm{C}(93)$.

The disc microenvironment is hostile. The avascular situation, nutrient deficiency, low oxygen concentration, increased acidity, and mechanical loading, all affects the 
survival, proliferation and differentiation of MSCs (94). Nutrient deprivation may present challenge but not a lethal threat to MSCs (95) (96). Hypertonicity, as well as increased acidity (95) (96), may inhibit the viability and matrix synthesis of MSCs. Despite the above, not all characteristics of IVD niche have bad influence for MSCs. Hypoxia is favorable for the chondrogenic differentiation of MSCs. Dynamic compression may promote NP-like differentiation (97), while radial compressive loadings may induce AF-like differentiation, in MSCs (98). Considering the timing for stem cell transplantation matters, that MSCs survives better in mild to moderate degenerated discs than severely degenerated ones (99), performing intervention of MSC implantation at earlier stage of disc degeneration may help to alleviate the stress of degenerated disc niche on MSCs and thus may promise a better clinical outcome.

\section{References}

1. Cheung, KM; Karppinen, J; Chan, D, et al. Prevalence and pattern of lumbar magnetic resonance imaging changes in a population study of one thousand forty-three individuals. Spine (Phila Pa 1976) 34(9):934-940; 2009.

2. Manchikanti, L; Singh, V; Pampati, V, et al. Evaluation of the relative contributions of various structures in chronic low back pain. Pain physician 4(4):308-316; 2001.

3. Fritzell, P; Hagg, O; Wessberg, P, et al. 2001 Volvo Award Winner in Clinical Studies: Lumbar fusion versus nonsurgical treatment for chronic low back pain: a multicenter randomized controlled trial from the Swedish Lumbar Spine Study Group. Spine (Phila Pa 1976) 26(23):2521-2532; discussion 2532-2524; 2001.

4. Fairbank, J; Frost, H; Wilson-MacDonald, J, et al. Randomised controlled trial to compare surgical stabilisation of the lumbar spine with an intensive rehabilitation programme for patients with chronic low back pain: the MRC spine stabilisation trial. BMJ 330(7502):1233; 2005.

5. Levin, DA; Hale, JJ; Bendo, JA. Adjacent segment degeneration following spinal fusion for degenerative disc disease. Bull NYU Hosp Jt Dis 65(1):29-36; 2007.

6. Longo, UG; Ripalda, P; Denaro, V, et al. Morphologic comparison of cervical, thoracic, lumbar intervertebral discs of cynomolgus monkey (Macaca fascicularis). Eur Spine J 15(12):1845-1851; 2006.

7. Bibby, SR; Urban, JP. Effect of nutrient deprivation on the viability of intervertebral disc cells. 
Eur Spine J 13(8):695-701; 2004.

8. LV, F; Leung, VY; Huang, S, et al. In search of nucleus pulposus-specific molecular markers. Rheumatology (Oxford) 53(4):600-610; 2014.

9. Maroudas, A; Stockwell, RA; Nachemson, A, et al. Factors involved in the nutrition of the human lumbar intervertebral disc: cellularity and diffusion of glucose in vitro. Journal of anatomy 120(Pt 1):113-130; 1975.

10. Stockwell, RA. The interrelationship of cell density and cartilage thickness in mammalian articular cartilage. Journal of anatomy 109(Pt 3):411-421; 1971.

11. Mwale, F; Roughley, P; Antoniou, J. Distinction between the extracellular matrix of the nucleus pulposus and hyaline cartilage: a requisite for tissue engineering of intervertebral disc. Eur Cell Mater 8:58-63; discussion 63-54; 2004.

12. Kuffler, DP; Sosa, IJ; Reyes, O. Schwann cell chondroitin sulfate proteoglycan inhibits dorsal root ganglion neuron neurite outgrowth and substrate specificity via a soma and not a growth cone mechanism. J Neurosci Res 87(13):2863-2871; 2009.

13. Melrose, J; Roberts, S; Smith, S, et al. Increased nerve and blood vessel ingrowth associated with proteoglycan depletion in an ovine anular lesion model of experimental disc degeneration. Spine (Phila Pa 1976) 27(12):1278-1285; 2002.

14. Junger, S; Gantenbein-Ritter, B; Lezuo, P, et al. Effect of limited nutrition on in situ intervertebral disc cells under simulated-physiological loading. Spine 34(12):1264-1271; 2009.

15. Soukane, DM; Shirazi-Adl, A; Urban, JP. Computation of coupled diffusion of oxygen, glucose and lactic acid in an intervertebral disc. J Biomech 40(12):2645-2654; 2007.

16. Hoyland, JA; Le Maitre, C; Freemont, AJ. Investigation of the role of IL-1 and TNF in matrix degradation in the intervertebral disc. Rheumatology (Oxford) 47(6):809-814; 2008.

17. Meir, A; McNally, DS; Fairbank, JC, et al. The internal pressure and stress environment of the scoliotic intervertebral disc--a review. Proceedings of the Institution of Mechanical Engineers. Part H, Journal of engineering in medicine 222(2):209-219; 2008.

18. Kim, KW; Chung, HN; Ha, KY, et al. Senescence mechanisms of nucleus pulposus chondrocytes in human intervertebral discs. Spine J 9(8):658-666; 2009.

19. Gruber, HE; Ingram, JA; Norton, HJ, et al. Senescence in cells of the aging and degenerating intervertebral disc: immunolocalization of senescence-associated beta-galactosidase in human and sand rat discs. Spine 32(3):321-327; 2007.

20. Kauppila, LI. Ingrowth of blood vessels in disc degeneration. Angiographic and histological studies of cadaveric spines. J Bone Joint Surg Am 77(1):26-31; 1995.

21. Aoki, Y; Akeda, K; An, H, et al. Nerve fiber ingrowth into scar tissue formed following nucleus pulposus extrusion in the rabbit anular-puncture disc degeneration model: effects of depth of puncture. Spine (Phila Pa 1976) 31(21):E774-780; 2006.

22. Freemont, AJ; Peacock, TE; Goupille, P, et al. Nerve ingrowth into diseased intervertebral disc in chronic back pain. Lancet 350(9072):178-181; 1997.

23. Antoniou, J; Steffen, T; Nelson, F, et al. The human lumbar intervertebral disc: evidence for changes in the biosynthesis and denaturation of the extracellular matrix with growth, maturation, ageing, and degeneration. J Clin Invest 98(4):996-1003; 1996.

24. Melrose, J; Smith, SM; Fuller, ES, et al. Biglycan and fibromodulin fragmentation correlates with temporal and spatial annular remodelling in experimentally injured ovine intervertebral 
discs. Eur Spine J 16(12):2193-2205; 2007.

25. Patel, KP; Sandy, JD; Akeda, K, et al. Aggrecanases and aggrecanase-generated fragments in the human intervertebral disc at early and advanced stages of disc degeneration. Spine (Phila Pa 1976) 32(23):2596-2603; 2007.

26. Anderson, DG; Li, X; Balian, G. A fibronectin fragment alters the metabolism by rabbit intervertebral disc cells in vitro. Spine (Phila Pa 1976) 30(11):1242-1246; 2005.

27. Horton, MR; Shapiro, S; Bao, C, et al. Induction and regulation of macrophage metalloelastase by hyaluronan fragments in mouse macrophages. J Immunol 162(7):4171-4176; 1999.

28. Peng, Y; LV, F-J. Symptomatic versus Asymptomatic Intervertebral Disc Degeneration: Is Inflammation the Key? Critical Reviews ${ }^{\mathrm{TM}}$ in Eukaryotic Gene Expression 25(1):13-21; 2015.

29. Malemud, CJ. Matrix metalloproteinases (MMPs) in health and disease: an overview. Front Biosci 11:1696-1701; 2006.

30. Vo, NV; Hartman, RA; Yurube, T, et al. Expression and regulation of metalloproteinases and their inhibitors in intervertebral disc aging and degeneration. Spine J 13(3):331-341; 2013.

31. Lv, F; Peng, Y; Lim, FL, et al. Matrix Metalloproteinase MMP12 is Associated with Intervertebral Disc Degeneration. Orthopaedic Research Symposium 2015 Annual Meeting. Las Vegas; 2015.

32. Richardson, SM; Doyle, P; Minogue, BM, et al. Increased expression of matrix metalloproteinase-10, nerve growth factor and substance $P$ in the painful degenerate intervertebral disc. Arthritis research \& therapy 11(4):R126; 2009.

33. Igarashi, T; Kikuchi, S; Shubayev, V, et al. 2000 Volvo Award winner in basic science studies: Exogenous tumor necrosis factor-alpha mimics nucleus pulposus-induced neuropathology. Molecular, histologic, and behavioral comparisons in rats. Spine (Phila Pa 1976) 25(23):2975-2980; 2000.

34. Ahn, SH; Cho, YW; Ahn, MW, et al. mRNA expression of cytokines and chemokines in herniated lumbar intervertebral discs. Spine (Phila Pa 1976) 27(9):911-917; 2002.

35. O'Donnell, JL; O'Donnell, AL. Prostaglandin E2 content in herniated lumbar disc disease. Spine (Phila Pa 1976) 21(14):1653-1655; discussion 1655-1656; 1996.

36. Shamji, MF; Setton, LA; Jarvis, W, et al. Proinflammatory cytokine expression profile in degenerated and herniated human intervertebral disc tissues. Arthritis and rheumatism 62(7):1974-1982; 2010.

37. Kang, JD; Stefanovic-Racic, M; Mclntyre, LA, et al. Toward a biochemical understanding of human intervertebral disc degeneration and herniation. Contributions of nitric oxide, interleukins, prostaglandin E2, and matrix metalloproteinases. Spine (Phila Pa 1976) 22(10):1065-1073; 1997.

38. Gilbertson, L; Ahn, SH; Teng, PN, et al. The effects of recombinant human bone morphogenetic protein-2, recombinant human bone morphogenetic protein-12, and adenoviral bone morphogenetic protein-12 on matrix synthesis in human annulus fibrosis and nucleus pulposus cells. Spine J 8(3):449-456; 2008.

39. Imai, Y; Miyamoto, K; An, HS, et al. Recombinant human osteogenic protein-1 upregulates proteoglycan metabolism of human anulus fibrosus and nucleus pulposus cells. Spine (Phila Pa 1976) 32(12):1303-1309; discussion 1310; 2007.

40. Wei, A; Williams, LA; Bhargav, D, et al. BMP13 prevents the effects of annular injury in an ovine model. Int J Biol Sci 5(5):388-396; 2009. 
41. Walsh, AJ; Lotz, JC. Biological response of the intervertebral disc to dynamic loading. J Biomech 37(3):329-337; 2004.

42. Abbott, RD; Purmessur, D; Monsey, RD, et al. Regenerative potential of TGFbeta3 + Dex and notochordal cell conditioned media on degenerated human intervertebral disc cells. J Orthop Res 30(3):482-488; 2012.

43. Gruber, HE; Norton, HJ; Hanley, EN, Jr. Anti-apoptotic effects of IGF-1 and PDGF on human intervertebral disc cells in vitro. Spine (Phila Pa 1976) 25(17):2153-2157; 2000.

44. Hayes, AJ; Ralphs, JR. The response of foetal annulus fibrosus cells to growth factors: modulation of matrix synthesis by TGF-beta1 and IGF-1. Histochem Cell Biol 136(2):163-175; 2011.

45. Pratsinis, H; Constantinou, V; Pavlakis, K, et al. Exogenous and autocrine growth factors stimulate human intervertebral disc cell proliferation via the ERK and Akt pathways. J Orthop Res 30(6):958-964; 2012.

46. Wallach, CJ; Sobajima, S; Watanabe, Y, et al. Gene transfer of the catabolic inhibitor TIMP-1 increases measured proteoglycans in cells from degenerated human intervertebral discs. Spine (Phila Pa 1976) 28(20):2331-2337; 2003.

47. Pratsinis, H; Kletsas, D. PDGF, bFGF and IGF-I stimulate the proliferation of intervertebral disc cells in vitro via the activation of the ERK and Akt signaling pathways. Eur Spine J 16(11):1858-1866; 2007.

48. Zara, JN; Siu, RK; Zhang, X, et al. High doses of bone morphogenetic protein 2 induce structurally abnormal bone and inflammation in vivo. Tissue Eng Part A 17(9-10):1389-1399; 2011.

49. Shen, B; Bhargav, D; Wei, A, et al. BMP-13 emerges as a potential inhibitor of bone formation. Int J Biol Sci 5(2):192-200; 2009.

50. Wehling, P; Schulitz, KP; Robbins, PD, et al. Transfer of genes to chondrocytic cells of the lumbar spine. Proposal for a treatment strategy of spinal disorders by local gene therapy. Spine (Phila Pa 1976) 22(10):1092-1097; 1997.

51. Liu, Y; Kong, J; Chen, BH, et al. Combined expression of CTGF and tissue inhibitor of metalloprotease-1 promotes synthesis of proteoglycan and collagen type II in rhesus monkey lumbar intervertebral disc cells in vitro. Chinese medical journal 123(15):2082-2087; 2010.

52. Liu, Y; Li, JM; Hu, YG. Transplantation of gene-modified nucleus pulposus cells reverses rabbit intervertebral disc degeneration. Chinese medical journal 124(16):2431-2437; 2011.

53. Le Visage, C; Kim, SW; Tateno, K, et al. Interaction of human mesenchymal stem cells with disc cells: changes in extracellular matrix biosynthesis. Spine (Phila Pa 1976) 31(18):2036-2042; 2006.

54. Masuda, K. Biological repair of the degenerated intervertebral disc by the injection of growth factors. Eur Spine J 17 Suppl 4:441-451; 2008.

55. Gorensek, M; Jaksimovic, C; Kregar-Velikonja, N, et al. Nucleus pulposus repair with cultured autologous elastic cartilage derived chondrocytes. Cell Mol Biol Lett 9(2):363-373; 2004.

56. Acosta, FL, Jr.; Metz, L; Adkisson, HD, et al. Porcine intervertebral disc repair using allogeneic juvenile articular chondrocytes or mesenchymal stem cells. Tissue Eng Part A 17(23-24):3045-3055; 2011

57. Erwin, WM; Islam, D; Inman, RD, et al. Notochordal cells protect nucleus pulposus cells from degradation and apoptosis: implications for the mechanisms of intervertebral disc 
degeneration. Arthritis Res Ther 13(6):R215; 2011.

58. Iwashina, T; Mochida, J; Sakai, D, et al. Feasibility of using a human nucleus pulposus cell line as a cell source in cell transplantation therapy for intervertebral disc degeneration. Spine (Phila Pa 1976) 31(11):1177-1186; 2006.

59. Nishimura, K; Mochida, J. Percutaneous reinsertion of the nucleus pulposus. An experimental study. Spine (Phila Pa 1976) 23(14):1531-1538; discussion 1539; 1998.

60. Nomura, T; Mochida, J; Okuma, M, et al. Nucleus pulposus allograft retards intervertebral disc degeneration. Clin Orthop Relat Res (389):94-101; 2001.

61. Okuma, M; Mochida, J; Nishimura, K, et al. Reinsertion of stimulated nucleus pulposus cells retards intervertebral disc degeneration: an in vitro and in vivo experimental study. J Orthop Res 18(6):988-997; 2000.

62. Gruber, HE; Ingram, JA; Davis, DE, et al. Increased cell senescence is associated with decreased cell proliferation in vivo in the degenerating human annulus. Spine J 9(3):210-215; 2009.

63. Richardson, SM; Mobasheri, A; Freemont, AJ, et al. Intervertebral disc biology, degeneration and novel tissue engineering and regenerative medicine therapies. Histol Histopathol 22(9):1033-1041; 2007.

64. Sheikh, H; Zakharian, K; De La Torre, RP, et al. In vivo intervertebral disc regeneration using stem cell-derived chondroprogenitors. J Neurosurg Spine 10(3):265-272; 2009.

65. Lv, FJ; Tuan, RS; Cheung, KM, et al. Concise review: the surface markers and identity of human mesenchymal stem cells. Stem Cells 32(6):1408-1419; 2014.

66. Quarto, R; Mastrogiacomo, M; Cancedda, R, et al. Repair of large bone defects with the use of autologous bone marrow stromal cells. N Engl J Med 344(5):385-386; 2001.

67. Horwitz, EM; Gordon, PL; Koo, WK, et al. Isolated allogeneic bone marrow-derived mesenchymal cells engraft and stimulate growth in children with osteogenesis imperfecta: Implications for cell therapy of bone. Proceedings of the National Academy of Sciences of the United States of America 99(13):8932-8937; 2002.

68. Tsai, TL; Nelson, BC; Anderson, PA, et al. Intervertebral disc and stem cells cocultured in biomimetic extracellular matrix stimulated by cyclic compression in perfusion bioreactor. Spine Journal: Official Journal of the North American Spine Society 14(9):2127-2140; 2014.

69. Lehmann, TP; Filipiak, K; Juzwa, W, et al. Coculture of human nucleus pulposus cells with multipotent mesenchymal stromal cells from human bone marrow reveals formation of tunnelling nanotubes. Mol Med Rep 9(2):574-582; 2014.

70. Watanabe, T; Sakai, D; Yamamoto, Y, et al. Human nucleus pulposus cells significantly enhanced biological properties in a coculture system with direct cell-to-cell contact with autologous mesenchymal stem cells. Journal of Orthopaedic Research 28(5):623-630; 2010.

71. Risbud, MV; Albert, TJ; Guttapalli, A, et al. Differentiation of mesenchymal stem cells towards a nucleus pulposus-like phenotype in vitro: implications for cell-based transplantation therapy. Spine 29(23):2627-2632; 2004.

72. Steck, E; Bertram, $\mathrm{H}$; Abel, $\mathrm{R}$, et al. Induction of intervertebral disc-like cells from adult mesenchymal stem cells. Stem Cells 23(3):403-411; 2005.

73. Richardson, SM; Walker, RV; Parker, S, et al. Intervertebral disc cell-mediated mesenchymal stem cell differentiation. Stem Cells 24(3):707-716; 2006.

74. Yuan, M; Yeung, CW; Li, YY, et al. Effects of nucleus pulposus cell-derived acellular matrix on 
the differentiation of mesenchymal stem cells. Biomaterials 34(16):3948-3961; 2013.

75. Yang, F; Leung, VY; Luk, KD, et al. Mesenchymal stem cells arrest intervertebral disc degeneration through chondrocytic differentiation and stimulation of endogenous cells. Mol Ther 17(11):1959-1966; 2009.

76. Sakai, D; Mochida, J; Iwashina, T, et al. Regenerative effects of transplanting mesenchymal stem cells embedded in atelocollagen to the degenerated intervertebral disc. Biomaterials 27(3):335-345; 2006

77. Crevensten, G; Walsh, AJ; Ananthakrishnan, D, et al. Intervertebral disc cell therapy for regeneration: mesenchymal stem cell implantation in rat intervertebral discs. Ann Biomed Eng 32(3):430-434; 2004.

78. Hiyama, A; Mochida, J; Iwashina, T, et al. Transplantation of mesenchymal stem cells in a canine disc degeneration model. J Orthop Res 26(5):589-600; 2008.

79. Henriksson, HB; Svanvik, $\mathrm{T}$; Jonsson, $\mathrm{M}$, et al. Transplantation of human mesenchymal stems cells into intervertebral discs in a xenogeneic porcine model. Spine (Phila Pa 1976) 34(2):141-148; 2009.

80. Haufe, SM; Mork, AR. Intradiscal injection of hematopoietic stem cells in an attempt to rejuvenate the intervertebral discs. Stem cells and development 15(1):136-137; 2006.

81. Coric, D; Pettine, K; Sumich, A, et al. Prospective study of disc repair with allogeneic chondrocytes presented at the 2012 Joint Spine Section Meeting. I Neurosurg Spine 18(1):85-95; 2013.

82. Meisel, HJ; Ganey, T; Hutton, WC, et al. Clinical experience in cell-based therapeutics: intervention and outcome. Eur Spine J 15 Suppl 3:S397-405; 2006.

83. Yoshikawa, $\mathrm{T}$; Ueda, $\mathrm{Y}$; Miyazaki, $\mathrm{K}$, et al. Disc regeneration therapy using marrow mesenchymal cell transplantation: a report of two case studies. Spine (Phila Pa 1976) 35(11):E475-480; 2010.

84. Orozco, L; Soler, R; Morera, C, et al. Intervertebral disc repair by autologous mesenchymal bone marrow cells: a pilot study. Transplantation 92(7):822-828; 2011.

85. Pettine, K; Suzuki, R; Sand, T, et al. Treatment of discogenic back pain with autologous bone marrow concentrate injection with minimum two year follow-up. International Orthopaedics; 2015.

86. Lv, F; Lu, M; Cheung, KM, et al. Intrinsic properties of mesemchymal stem cells from human bone marrow, umbilical cord and umbilical cord blood comparing the different sources of MSC. Curr Stem Cell Res Ther 7(6):389-399; 2012.

87. Huang, YZ; Cai, JQ; LV, FJ, et al. Species variation in the spontaneous calcification of bone marrow-derived mesenchymal stem cells. Cytotherapy 15(3):323-329; 2013.

88. Lv FJ ; Sun Y ; Zhou LX, et al. The Potential of Umbilical Cord Derived Mesenchymal Stem Cells in Intervertebral Disc Repair. Global Spine Journal 4(S 01):S81-82; 2014.

89. Sakai, D; Mochida, J; Yamamoto, Y, et al. Transplantation of mesenchymal stem cells embedded in Atelocollagen gel to the intervertebral disc: a potential therapeutic model for disc degeneration. Biomaterials 24(20):3531-3541; 2003.

90. Gaetani, P; Torre, ML; Klinger, M, et al. Adipose-derived stem cell therapy for intervertebral disc regeneration: an in vitro reconstructed tissue in alginate capsules. Tissue Eng Part A 14(8):1415-1423; 2008.

91. Nesti, Lj; Li, WJ; Shanti, RM, et al. Intervertebral disc tissue engineering using a novel 
hyaluronic acid-nanofibrous scaffold (HANFS) amalgam. Tissue Eng Part A 14(9):1527-1537; 2008.

92. Richardson, SM; Hughes, N; Hunt, JA, et al. Human mesenchymal stem cell differentiation to NP-like cells in chitosan-glycerophosphate hydrogels. Biomaterials 29(1):85-93; 2008.

93. Wiltsey, C; Kubinski, P; Christiani, T, et al. Characterization of injectable hydrogels based on poly(N-isopropylacrylamide)-g-chondroitin sulfate with adhesive properties for nucleus pulposus tissue engineering. J Mater Sci Mater Med 24(4):837-847; 2013.

94. Huang, YC; Leung, VY; Lu, WW, et al. The effects of microenvironment in mesenchymal stem cell-based regeneration of intervertebral disc. Spine J 13(3):352-362; 2013.

95. Liang, C; Li, H; Tao, Y, et al. Responses of human adipose-derived mesenchymal stem cells to chemical microenvironment of the intervertebral disc. J TransI Med 10:49; 2012.

96. Wuertz, K; Godburn, K; Neidlinger-Wilke, C, et al. Behavior of mesenchymal stem cells in the chemical microenvironment of the intervertebral disc. Spine (Phila Pa 1976) 33(17):1843-1849; 2008

97. Dai, J; Wang, H; Liu, G, et al. Dynamic compression and co-culture with nucleus pulposus cells promotes proliferation and differentiation of adipose-derived mesenchymal stem cells. J Biomech 47(5):966-972; 2014.

98. See, EY; Toh, SL; Goh, JC. Effects of radial compression on a novel simulated intervertebral disc-like assembly using bone marrow-derived mesenchymal stem cell cell-sheets for annulus fibrosus regeneration. Spine (Phila Pa 1976) 36(21):1744-1751; 2011.

99. Ho, G; Leung, VY; Cheung, KM, et al. Effect of severity of intervertebral disc injury on mesenchymal stem cell-based regeneration. Connect Tissue Res 49(1):15-21; 2008. 